\title{
LAS RENTAS AGRARIAS Y RURALES EN EXTREMADURA
}

\section{J. L. Gurría Gascón ${ }^{1}$, A. Nieto Masot ${ }^{1}$ y A. Hernández Carretero²}

1 Dpto. de A. y Ciencias del Territorio. Facultad de Filosofía y Letras 2 Dpto. de Didáctica de las Ciencias Sociales. Facultad de Formación del Profesorado.

Campus Universitario de Cáceres

jlgurria@unex.es; ananieto@unex.es; ahernand@unex.es

\begin{abstract}
Resumen: Extremadura es una región eminentemente rural, por cuanto que las veinte ciudades principales tan sólo aglutinan a la mitad de la población. En las últimas dos o tres décadas se han producido profundas transformaciones que han supuesto el paso de una economía agraria de subsistencia a una economía competitiva de los servicios, que ha afectado tanto a las ciudades como, de manera más atenuada, a los ámbitos rurales. Estos cambios, no obstante, no han sido suficientes para explicar la estabilidad de la población en los núcleos rurales, todavía bastante dependientes de un sector agrario incapaz de impulsar el desarrollo de pueblos y comarcas por sí solo. Sin embargo, al amparo de las coyunturas de las últimas décadas, la población rural ha sabido generar una serie de rentas mixtas multisectoriales y nuevas dinámicas, basadas en la movilidad, que han permitido un nivel de vida digno y la estabilidad de su población.
\end{abstract}

Palabras clave: Espacios Rurales, Rentas, Desarrollo Económico, Extremadura.

\begin{abstract}
Extremadura is a largely rural region, in fact twenty largest cities agglutinate only half of the population. In the last two or three decades profound changes have occurred which marked the transition from a subsistence agricultural economy to a competitive service economy, which has affected both the cities and, more attenuated, the rural areas. These changes, however, were not sufficient to explain the stability of the population in rural areas, still quite dependent on the agricultural sector unable to produce the development of these areas.
\end{abstract}

Recibido: 14-3-11. Aceptado: 2-2-12. 
However, under the politics and economic situation of the last decades, the rural population has managed to generate a series of mixed multisectoral income and new dynamics, based on mobility, which allowed an increase of level of living and the population stability.

Keywords: Rural Areas, Incomes, Economic Development, Extremadura.

\section{Introducción y objetivos}

Asociadas a las profundas transformaciones socioeconómicas de las últimas décadas, las familias rurales se han ido adaptando con rapidez a las nuevas coyunturas, a través de un sistema de rentas multisectoriales y complementarias, de dentro y de fuera, procedentes de los tres sectores de actividad y de otras fuentes pasivas. Esto ha permitido el incremento de los ingresos en el medio rural y, en definitiva, la estabilización de su población hasta en los núcleos más pequeños, como norma general.

El sector agrario, tradicional dedicación del mundo rural, es cada vez más secundario y a tiempo parcial, con un acelerado descenso del empleo y de la actividad, siendo muy pocas las familias con rentas procedentes de este sector en exclusiva o como actividad principal.

Sin embargo, en su conjunto, no se puede constatar, que se hayan desarrollado de manera paralela los otros sectores de actividad, lo que no deja de ser un tanto contradictorio con su supuesta y aceptada terciarización y con la complementariedad de las rentas provenientes del resto de sectores de actividad.

El paro agrario y el envejecimiento son las notas más definitorias, en las últimas décadas, del medio rural extremeño, pero las rentas pasivas derivadas de estos subsidios y pensiones no son suficientes, junto a las provenientes de Europa (PAC y Programas de Desarrollo Rural), para explicar el incremento de las rentas.

Nuestro objetivo, en primer lugar, es el conocimiento de las rentas emanadas del medio rural, con el fin de demostrar que son insuficientes, en sí mismas, para dar explicación al incremento de los ingresos y a la estabilización de la población. En segundo lugar, confirmar que la población rural se ha terciarizado, pero no los ámbitos rurales, sobre la base de la creciente movilidad laboral de la población rural hacia los núcleos urbanos próximos. Y, en tercer lugar y en relación con los objetivos anteriores, la constatación de las nuevas dinámicas territoriales, aspecto fundamental para la planificación futura del desarrollo rural. 
No existen datos disponibles para el estudio de estas rentas en la región y la literatura es muy exigua, por lo que es necesario recurrir a numerosas fuentes, más o menos indirectas.

Las fuentes utilizadas provienen de la Encuesta de Población Activa (INE), de la Encuesta de Estructura Salarial (INE), de la Encuesta de Presupuestos Familiares (INE) y del Anuario de Estadísticas Laborales y de Asuntos Sociales (Ministerio de Trabajo y Asuntos Sociales). Para las rentas agrarias, se ha utilizado, sobre todo, la Encuesta sobre la Estructura de las Explotaciones Agrícolas (INE, 2007 y Consejería de Agricultura, 2010).

\section{Las rentas agrarias y rurales}

Extremadura, como región agraria y rural secularmente, ha venido ocupando los últimos lugares en niveles de renta a escala nacional de manera persistente hasta la actualidad, como consecuencia de un desajuste permanente entre la población y los recursos, que se han traducido en una intensa emigración y, posteriormente, en elevados índices de paro. Como indicaba Frutos (1979: 8-12): El sector primario es boy esencial para Extremadura. Pero constituye a un tiempo su riqueza y su pobreza... De becho, el predominio de la agricultura y la ganadería forman parte de un patrimonio de pobreza que Extremadura arrastra desde el medioevo, y que pervive hoy porque perviven similares estructuras... Pero si la producción agropecuaria debería ser la que apoyase el desarrollo regional, de hecho no es sino un foco más de depresión...

En las tres últimas décadas, sin embargo, la situación ha cambiado sustancialmente, aunque la población rural, sobre todo la que mantiene una mayor dependencia del sector agrario, se sigue debatiendo entre la subsistencia y la competitividad, con fuertes restricciones, impuestas tanto por las limitaciones naturales como por las estructurales.

En estos momentos, parte de la población rural mantiene sus comportamientos de subsistencia, aunque sobre bases totalmente diferentes: aprovechan los recursos agrarios con otras rentas pasivas, que complementan con el resto de los sectores de actividad, dentro o fuera. Como se indica en el Informe de TESYT (1991: 172-173): ... un elevado número de familias que... nutren la economía familiar de fuentes muy diversas y en su práctica totalidad irregulares e incontables.

La población rural ha conseguido conformar unas rentas multisectoriales, que permiten la subsistencia e, incluso el ahorro, sobre la base de un nivel de autoconsumo todavía elevado y un gasto reducido, lo que garantiza un bienestar y un nivel de vida digno en los núcleos rurales. Pero, en cualquier caso, son economías muy precarias, 
puesto que cualquier acción puede ocasionar la ruptura de esta organización socioeconómica y el abandono de los núcleos, probablemente definitivo.

La complejidad es mucho mayor si se considera que buena parte de estas rentas se obtienen fuera de los núcleos rurales, en las cabeceras comarcales y ciudades próximas y, en buena medida, a través de una economía sumergida, que el INE estima en torno al $20 \% 0 \%$.

Las transformaciones de las últimas décadas han permitido la convergencia más rápida de la región con las medias nacionales. Ha sido lenta, pero sostenida, muy importante si se consideran los obstáculos de partida y las dificultades para superar las múltiples limitaciones estructurales que todavía arrastra esta economía. Es una evolución que está muy correlacionada con la lenta desagrarización y con el creciente y correlativo proceso de terciarización.

Por otra parte, los Fondos Estructurales y los provenientes de la PAC y de los Programas de Desarrollo Rural también han contribuido a la modernización de las infraestructuras productivas y sociales, a la dinamización socioeconómica y al incremento de las rentas regionales y rurales.

Este crecimiento viene a explicar otros tres fenómenos no menos relevantes: la desaparición de los movimientos emigratorios, el fuerte descenso del paro en las décadas de final y de principio de siglo a pesar de los constantes excedentes agrarios, y la estabilización de la población en la región y en los ámbitos rurales.

\subsection{Estructura sectorial del empleo y de las rentas: el sector agrario}

Dentro de la estructura sectorial, destaca la reducida generación de empleo del sector agrario (11'4\%), en continuo descenso, y del industrial (8'5\%), con una aportación tan sólo del 8'4 y del 10’2\% respectivamente al VAB. La construcción, por su parte, sigue con el pujante desarrollo que la ha caracterizado en la última década, dando empleo al $15^{\prime} 5 \%$ del total y aportando al VAB el 16'1\%, casi tanto como los dos sectores anteriores conjuntamente. En estos dos últimos años de crisis económica, este subsector ha sido de los más afectados, habiendo caído casi cinco puntos hasta finales de 2010 .

El INE (2009), en la Encuesta Anual de Estructura Salarial, que no contempla el sector agrario, asigna a la industria el salario más elevado, con 19.000 Euros; los Servicios, con 18.500; y la construcción, con 16.200; salarios todos ellos por encima de la media regional, que se sitúa en los 16.000.

El sector agrario, tan sólo tiene un coste salarial del 2'5\% del total regional. Esto supone unos ingresos por asalariado y año de 4.344 Euros únicamente. Es un empleo 
muy estacional y mal remunerado, en definitiva un subempleo crónico con sueldos misérrimos.

Con este gasto total en salarios, el sector agrario sólo podría dar cabida a 10.827 empleos, en lugar de los 48.200 que tiene, para alcanzar los salarios medios de la construcción, que son los más bajos tras los agrarios. Y, probablemente, éstos puedan ser los excedentes poblacionales en los próximos años, además de los pequeños empresarios, que no podrán competir. Dicho de otro modo, con este coste salarial, los 48.200 empleados agrarios, la mayoría jornaleros eventuales, tendrían trabajo durante 2'7 meses al año para alcanzar el salario medio de la construcción en estos meses.

La Encuesta de Estructura de las Explotaciones Agrícolas (INE, 2008 y Consejería de Agricultura y Desarrollo Rural, 2010) indica que se dedican al sector agrario, como actividad exclusiva, 46.000 titulares de explotaciones, 33.542 cónyuges y 8.057 familiares. Hay un claro predominio de la mano de obra parental, lo que indica el predominio de la pequeña explotación.

Por ello, 3.571 titulares tienen otra actividad secundaria complementaria, al igual que 735 cónyuges y 2.455 familiares, de tal manera que, dentro de la unidad familiar, 6.761 personas aportan otras rentas complementarias procedentes de otras actividades secundarias.

Otros 16.978 titulares, junto a 10.605 cónyuges y 15.496 familiares, deben de recurrir a otra actividad principal, con una dedicación agraria a tiempo parcial o como actividad muy secundaria, muchas veces para seguir percibiendo pequeñas subvenciones comunitarias.

En el cómputo total, obtienen ingresos de otras actividades fuera del sector agrario, bien sea como actividad principal o secundaria, 20.549 titulares de explotaciones, una tercera parte del total, además de 11.340 cónyuges y de 17.951 familiares, normalmente hijos.

El total de personas con alguna dedicación agraria asciende a 137.546, de los cuales obtienen ingresos complementarios de otras actividades 57.897 , lo que representa el 42 '0\%.

Además de los titulares de explotaciones y sus familiares, existen otros 48.200 asalariados, de los cuales una tercera parte aproximadamente son fijos y el resto eventuales. En uno y otro caso, los salarios son muy bajos e insuficientes para la subsistencia.

Los trabajadores fijos suelen ocupar puestos de guardería o cuidado del ganado, con sueldos de auténtica subsistencia. En cuanto a los eventuales, casi todo lo que 
perciben proviene del subsidio de desempleo agrario, ingresando por ello 2.526 Euros/año.

La Encuesta sobre la Estructura de las Explotaciones Agrícolas (2008) las clasifica según las Unidades de Dimensión Europea (UDE=1.200 Euros de margen bruto).

En 2007, el 50'0\% de las explotaciones agrarias no alcanza las cuatro UDEs, lo que las hace totalmente inviables, y otro 20 '0\% tenía entre 4 y 12 , lo que supone un margen bruto de ingresos entre 4.800 y 14.400 Euros anuales. Este margen equivaldría, para una familia media de 2'76 miembros, a unas rentas per cápita entre 1.739 y 5.217 Euros anuales. Esto evidenciaría que el 70 '0\% de las explotaciones agrarias tendrían una renta per cápita inferior al 70’0\%, como mínimo, de la media regional. Son, por lo tanto, explotaciones inviables sin otros ingresos procedentes del propio sector o de otros sectores económicos.

Únicamente el 15\%\% de las explotaciones estaría por encima de las 40 UDEs, umbral mínimo para que una familia pudiera alcanzar las rentas medias regionales. Y sólo el 7’7\% tendría más de 60 UDEs. Estas últimas alcanzarían unas rentas per cápita entre el 61'0 y el 167'0\% por encima de las medias regionales. Por otra parte, hay que destacar que este porcentaje de explotaciones mayores acapara también en torno al $65^{\prime} 0 \%$ de todas las subvenciones de la PAC, lo que viene a constatar la permanencia de la dicotomía y la estabilidad de la estructura de la propiedad y de las explotaciones desde mediados del siglo XX al menos, según los datos disponibles.

La situación de la población dependiente del sector agrario, tanto de los jornaleros como de los pequeños propietarios, es bastante crítica y, desde luego, insuficiente como actividad en exclusiva o como actividad principal. Necesitan, en consecuencia, recurrir a otras fuentes, tanto activas como pasivas, para conseguir unas rentas que, al menos, les permitan una vida digna. En este sentido, García Sanz (1999: 252) indica que hay una parte importante de los ingresos de los hogares llamados agrarios que no procede de la agricultura.

Y, más específicamente, TESYT (1991: 172-173) puntualiza que ...el padre, parado por supuesto, que hace chapuzas o se dedica a acarrear y vender leña en un pequeño furgón durante el invierno; los bijos baciendo unos jornales aquí y allá, como porteadores de un camión o jornadas en la construcción que complementan con el PER, y en algunas épocas del año andan a la recogida y venta de bortalizas silvestres (trigueros, cardillos, criadillas...); la madre haciendo horas en algunas casas; alguna bija de externa o interna en alguna casa...

También merecen una consideración especial, dentro del sector agrario, las subvenciones de la PAC, que aportan 622 millones de Euros anuales, nada menos que un $43^{\prime} 0 \%$ de las rentas agrarias, lo que indica la baja productividad del sector en su 
conjunto. En relación con la distribución de las mismas, sólo el 7’0\% de los empresarios agrarios percibirían, a tenor de la estructura de las explotaciones, el 65'0\% del total, lo que equivaldría a unas percepciones per cápita de 46.000 Euros. Por el contrario, el 93'0\% de pequeños y medianos empresarios únicamente percibirían una media de 1.873 Euros per cápita, lo que indica la inviabilidad de estas explotaciones, aun con las percepciones de la PAC. Sin embargo, todos estos ingresos sí son importantes en un sistema de rentas sectoriales complementarias, tanto entre los jornaleros como entre los pequeños y medianos empresarios.

Con un sector agrario de estas características, que sigue ocupando a un 33'0\% de la población activa en los núcleos con menos de 5.000 habitantes y a más de un $65^{\circ} 0 \%$ en los núcleos de menos de 2.000, es mucho más necesaria la diversificación de las actividades, del empleo y de las rentas fuera de este sector, que además se encuentra en franca regresión y cada vez con menor empleo. Así lo manifiesta García Sanz (2002: 3), al afirmar que la experiencia de los últimos años nos está indicando que se puede dar un desarrollo rural cada vez con menos bases agrarias y, más adelante, añade que las zonas rurales demográficamente más positivas son aquellas que han sabido avanzar en los procesos de diversificación ocupacional, entre los que tienen un puesto destacado los servicios, pero también, la industria agroalimentaria.

En cuanto a las rentas medias per cápita, la población dependiente del sector agrario (125.143 personas), dispondría de una renta en torno a los 10.500 Euros, tan sólo en torno al 65'0\% de la renta media extremeña. Con estos ingresos, podrían alcanzar las rentas medias regionales 82.069 personas, en el supuesto de que se distribuyeran por igual entre toda la población agraria. Pero la distribución de las explotaciones no guarda esta uniformidad y sigue manteniendo una fuerte dispersión de las rentas.

\subsection{Las rentas pasivas: subsidio agrario y pensiones}

Hay que considerar, por otra parte, que de los 48.200 asalariados, unos 21.000 tienen prestaciones del subsidio agrario durante seis meses al año, con un ingreso neto mensual de 421 Euros (505 Euros brutos, menos 83 Euros del "sello" de la cotización a la Seguridad Social), que supone 63'6 millones de Euros anuales, un 23'3\% de los costes salariales del sector.

Teniendo en cuenta los costes salariales y el subsidio agrario, el total anual se elevaría a 273’004 millones de Euros, por lo que las percepciones económicas brutas serían de 5.664 Euros por asalariado agrario.

Evidentemente, con estos ingresos difícilmente puede vivir una persona y menos una familia, aun en el caso de que pueda haber dos y tres perceptores del subsidio 
agrario en el seno de la misma. Está claro que deberán recurrir a otros recursos en otros sectores de actividad para complementar estos míseros ingresos y garantizar su subsistencia al menos. Por otra parte, este subsidio está promoviendo una economía sumergida, que favorece tanto a los asalariados agrarios como a los empresarios de todos los sectores, pues estos subsidiados únicamente pueden trabajar sin contratos para seguir percibiendo el subsidio agrario.

Aunque este fraude económico está cada vez más constatado en todos los ámbitos y sectores, está más extendido en el medio rural de Extremadura y Andalucía por las razones mencionadas. El Ministerio de Trabajo, en 2003, hizo aflorar 2.675 empleos ocultos en Extremadura, la mayoría en el sector agrario y, especialmente, entre el colectivo de inmigrantes, con o sin papeles.

Por estas razones, es lógico que en Extremadura en su conjunto y, más aún, en el medio rural, las rentas sean bajas, dado que existen una serie de factores estructurales y discriminantes, muy persistentes, que impiden una convergencia mayor con las medias regionales y nacionales.

Como afirma García Sanz (1999) estas diferencias están más acentuadas en Extremadura, las dos Castillas, Aragón, Andalucía, etc., como consecuencia del mayor peso del paro agrario y del envejecimiento.

Los núcleos rurales han seguido un proceso de envejecimiento persistente desde comienzos de los años setenta hasta la actualidad, como consecuencia de la emigración de las décadas de los sesenta y setenta y la mayor esperanza de vida.

En la región, la Seguridad Social gestiona 205.888 pensiones (enero 2010), con una percepción media de 645'1 Euros/mes, un 84’9\% de la pensión media nacional. Esto supone unas rentas que son inferiores, como mínimo, en un 40'0\% a las rentas medias per cápita regionales. En 2001, Extremadura era la penúltima región según la percepción media de las pensiones contributivas, con 57.000 pesetas mensuales. A finales de 2009, EP/Agencias titulaba Extremadura a la cola en cuanto al importe de las pensiones. Para el último año, el Ministerio de Trabajo (2010) facilita las prestaciones por jubilación que se recogen en la Tabla 1.

Tabla 1. Prestaciones por jubilación.

\begin{tabular}{|lccc|}
\hline \multicolumn{1}{|c}{ Régimen } & Total jubilaciones & Cuantía mensual & Cuantía anual \\
\hline General & 95.598 & 767,63 & $10.746,82$ \\
Autónomos & 50.984 & 534,45 & $7.482,30$ \\
Régimen Especial Agrario & 45.302 & 539,50 & $7.553,00$ \\
Otras & 13.506 & 600,10 & $8.401,40$ \\
No contributivas & 15.000 & 339,70 & $4.755,80$ \\
\hline
\end{tabular}

Fuente: Ministerio de Trabajo: Estadísticas e Informes de la Seguridad Social y elaboración propia. 
Este volumen de jubilados, junto a sus bajas pensiones, está hipotecando el crecimiento de las rentas regionales de manera permanente. La causa fundamental se debe a que, entre los jubilados, el mayor porcentaje proviene de los empresarios autónomos y del Régimen Especial Agrario, con unas prestaciones un 18'0\% inferiores a la media por jubilación. En el caso de los autónomos, el 84’0\% ha cotizado el valor mínimo a la Seguridad Social, al tratarse de pequeños empresarios (Asociación de Trabajadores Autónomos, ATA). Entre los procedentes del sector agrario, el pago de los "sellos" a la Seguridad Social también es reducido, muchos de ellos tienen pocos años cotizados e, incluso, no han llegado a cotizar nunca, de ahí que buena parte de las prestaciones no contributivas se deban a ello o que, en los últimos años, el mayor crecimiento del paro agrario se está produciendo entre los hombres y mujeres por encima de los 55 años, con esta misma finalidad.

En definitiva, Extremadura, las dos Castillas y Aragón son las Comunidades con una tasa de población dependiente más elevada, ya que rebasan el 50'0\%. Hay que destacar, además, que del total de jubilados, en la región únicamente perciben una pensión menos del 57’0\%, frente al 67\%6\% de la media nacional (La Caixa, 2000).

Extremadura es la de menor porcentaje de perceptores, junto con Canarias y Castilla-La Mancha, y, evidentemente, los ámbitos más afectados son los rurales y, dentro de ellos, los jubilados agrarios. De hecho, de los jubilados extremeños, la mitad se encuentran en municipios con menos de 5.000 habitantes, y en casi la mitad de los hogares extremeños existe alguna persona mayor de 65 años.

García Sanz (1999) indicaba, en definitiva, que los pensionistas rurales, tenían un $18^{\prime} 0 \%$ menos de percepciones que los urbanos, al igual que ocurre con los inactivos $\left(-19^{\prime} 0 \%\right)$, con las amas de casa $\left(-22^{\prime} 0 \%\right)$, con los varones rurales $\left(-200^{\prime} 0 \%\right)$, sobre todo los agrarios, y con los perceptores del subsidio de desempleo (-27'0\%), especialmente los jornaleros eventuales agrarios. Son éstas, precisamente, las características demográficas predominantes en los ámbitos rurales, de ahí sus bajas rentas.

\subsection{Los ingresos y gastos de los hogares}

Otra aproximación a las rentas rurales se puede realizar a través del gasto, según los datos aportados por la Encuesta de Presupuestos Familiares (INE, 2008).

En la región, el gasto medio por persona es de 9.146 Euros, que supone el 56'8\% de la renta media per cápita, un gasto reducido, que se explica por el más bajo nivel de vida en los ámbitos rurales y en la región, el menor consumo de las personas mayores, las mentalidades menos consumistas de la población rural y su mayor nivel de autoconsumo, la disponibilidad de viviendas en los núcleos rurales, etc., si bien en las dos últimas décadas han ido cambiando progresivamente estos hábitos de con- 
sumo, equiparándose progresivamente a los urbanos. Sobre la media nacional, este gasto es del $77^{\prime} 5 \%$, un porcentaje, no obstante, ligeramente superior al de las rentas medias.

El gasto medio por hogar es de 25.280 Euros y, teniendo en cuenta que la composición familiar media es de 2'76 miembros, el problema es que estos gastos son un 58 '0\% superiores a los ingresos de un solo salario medio. Es necesario, como mínimo, más de un empleo y medio por hogar sólo para cubrir los gastos anuales, de ahí la necesidad de que trabajen dos miembros en cada unidad familiar, con las limitaciones del reducido empleo femenino y joven, que retrasa y dificulta la incorporación al mundo laboral de estos segmentos poblacionales, reduce el consumo y la posibilidad de emancipación o de formación de una familia, etc.

En cuanto a los gastos según la fuente principal de ingresos, sólo el 16’7\% son de personas que trabajan por cuenta propia o disponen de rentas de la propiedad y del capital, que es el segmento de mayor gasto por hogar (40.136 Euros), casi el doble que el segmento poblacional de ingresos pasivos, que alcanza únicamente el 25'8\% del gasto regional.

Son referencias de lo que puedan suponer estos ingresos pasivos en la conformación de las rentas regionales, aunque hay que tener en cuenta que en los ámbitos rurales, con casi una tercera parte de personas mayores y el 43'0\% de subsidiados sobre el total del empleo agrario, estos ingresos pasivos pueden oscilar entre el 40'0 y el $60 \%$ del total de las rentas.

Los hogares con menos de mil euros de ingresos mensuales, que suponen el 33'0\% de los hogares, gastan el 21'2\% del total regional, con un gasto por persona del 89'0\% de la media extremeña. Suelen ser hogares de una o dos personas, normalmente mayores. El 43'0\% de los hogares ingresan menos de 2.000 Euros mensuales, con una participación similar en los gastos, lo que los equipara con las medias del gasto por persona y hogar. En cambio, los hogares con más de 3.000 Euros mensuales de ingresos tan sólo representan el 6'8\% del total y gastan el 13'3\%, con una media de 49.373 Euros, casi el doble que la media regional.

Los gastos principales se concentran en la vivienda (28'4\%), y en alimentación y artículos de vestir (23'1\%). Lo que se pueden considerar como gastos básicos, apenas rebasan el 50'0\%, que es una cantidad baja, tanto por la mayor disponibilidad de viviendas en propiedad, como por el mayor nivel de autoconsumo, sobre todo en los pueblos. Es muy representativo también el gasto en los transportes, que alcanza el $16{ }^{\prime} \%$, un gasto elevado, que indica el grado de movilidad de la población. Finalmente, el otro capítulo más destacable es el del ocio, cafés y restauración, con un 153\%, que viene a expresar el grado de consumismo y el desarrollo del sector servicios, que tiene bastante que ver con estos comportamientos. 


\section{La movilidad rural}

En los núcleos rurales no existe mucho empleo fuera del sector agrario, pero en las dos últimas décadas se han generado nuevas dinámicas, que han permitido la complementariedad de rentas mixtas o multisectoriales: la acción de los Grupos de Acción Local (GAL) para la gestión de los Programas de Desarrollo Rural, el turismo rural, las segundas residencias, la inmigración de autóctonos y de foráneos más recientemente, entre ellos los neorrurales, etc., pero sobre todo los movimientos pendulares de trabajadores en ambas direcciones, básicamente hacia las ciudades vecinas. Todo ello se inserta bajo el concepto de la movilidad, que -de acuerdo con Módenes (2006) - se ha convertido en un factor estructurante del territorio y de la sociedad actual. En términos similares se expresa Oliva (2006: 144), al afirmar que la itinerancia es ya una forma intrínseca de la vida rural para muchos grupos sociales.

Esta movilidad ha permitido una mayor diversificación del empleo, la complementariedad de rentas y, en alguna medida, la incorporación de la mujer y de los jóvenes al mundo laboral. Las ciudades y la accesibilidad han jugado, en este sentido, un papel esencial en sus entornos rurales, favoreciendo la diversificación a través de los movimientos en ambos sentidos. Como indica Oliva (2006: 158-160), el 70'0\% de los jóvenes y el 66’0\% de las mujeres se desplazan diariamente desde los núcleos más pequeños hasta las ciudades por razones laborales.

Efectivamente, Camarero (2009: 165) señala que ...los mercados de trabajo rurales presentan una fragilidad que, a menudo, sólo puede ser superada mediante la movilidad.

En Extremadura, los trabajadores foráneos en los núcleos rurales presentan fuertes oscilaciones, en función sobre todo del empleo ofertado a nivel local y de la accesibilidad, con valores que van desde el 10'0 al 40'0\% de la población ocupada. Sin embargo, la movilidad exterior hacia las ciudades es mucho más intensa y regular, dependiendo igualmente de la proximidad y accesibilidad de los núcleos urbanos, de su tamaño y de la oferta laboral, oscilando entre un 25'0 y un 45'0\% de la población ocupada de los núcleos rurales (Pérez, J.A. y Gurría, J.L., 2010: 57 y sig.).

La ciudad, por lo tanto, está ejerciendo una influencia predominante en el desarrollo rural y en la estabilización de su población a través de las nuevas dinámicas territoriales generadas en las tres últimas décadas. La propia UE, en la Estrategia Territorial Europea (ETE, 1999), ya es consciente de la importancia del papel de las ciudades en este sentido, llegando a vincular el desarrollo rural y el de sus núcleos rurales con la proximidad a las ciudades, al igual que hace en el Tercer Informe sobre la Cohesión Económica y Social (Gurría, 2007). En este sentido, Extremadura dispone de un sistema policéntrico de ciudades, bien distribuidas en el territorio, de tal 
manera que en el entorno de los $30 \mathrm{Km}$ de las principales ciudades y cabeceras comarcales se concentra el 88'1\% de la población regional, lo que ha favorecido, de manera espontánea, la consolidación de estas nuevas dinámicas territoriales, el desarrollo rural y la estabilización de su población.

\section{Conclusiones}

Las fuentes oficiales confirman la creciente desagrarización de los ámbitos rurales y, correlativamente, una importante terciarización de los mismos, pero al vincular la actividad a las personas y no a los lugares donde se desarrolla esta actividad, han inducido a frecuentes interpretaciones erróneas. Es cierto que se ha producido una cierta terciarización en los núcleos rurales, bastante limitada, así como una incipiente industrialización, al amparo de los Programas de Desarrollo Rural, pero no en la medida que parecen indicar las estadísticas disponibles.

Los núcleos rurales siguen estando todavía muy dependientes de un sector agrario muy desequilibrado y en declive, y de unas rentas pasivas provenientes de Europa, de las pensiones y del subsidio agrario, que no pueden garantizar el desarrollo socioeconómico por sí solas. De ahí la precariedad de estas economías, la inestabilidad de su población y la necesidad de buscar otras rentas complementarias en el resto de los sectores de actividad en las ciudades próximas.

Los núcleos rurales han encontrado en la movilidad laboral la posibilidad de una diversificación del empleo y de las rentas fuera del sector agrario, sin necesidad de emigrar, como ocurría en el pasado, conformado un conjunto de rentas mixtas y complementarias, que han permitido la estabilidad de la población. Esto se ha visto favorecido por un sistema urbano policéntrico en la región, que se erige en la actualidad en el principal factor del desarrollo rural en Extremadura.

\section{Bibliografía}

Bericat, E. (1994). Sociología de la movilidad espacial. El sedentarismo nómada. Madrid, Centro de Investigaciones Sociológicas.

Caixa, La (2000). Informe Social de España, 2000. Barcelona.

Camarero, L. (2006). Mercados de trabajo rurales: notas y reflexiones. Revista Española de Estudios Agrosociales y Pesqueros, 211, p. 11-18.
Frutos, L. M. (1979). Indicativos de subdesarrollo en Extremadura, F.n. Estudios dedicados a Carlos Callejo Serrano. Cáceres, Excma. Diputación Provincial.

García Sanz, B. (2003). Sociedad rural y desarrollo. Madrid, Ministerio de Agricultura, Pesca y Alimentación.

Gurría, J.L. (2007). El sistema urbano en Extremadura y su influencia en el 
desarrollo rural. Ciudad y Territorio. Estudios Territoriales, XXXIX, 151, p. 5382.

Gurría, J.L., Nieto, A. y Hernández, A.M ${ }^{\mathrm{a}}$ (2009). The new rural dynamics in southern Spain (Extremadura). F.n. Frutos, L.M ${ }^{\mathrm{a}}$, Climent, E. y Ruiz, E. New ruralities and sustainable use of territory. Zaragoza, Universidad de Zaragoza y CSRS-IGU, p. 383-404.

INE (2008). Encuesta de Presupuestos Familiares. Madrid.

INE (2008). Encuesta sobre la Estructura de las Explotaciones Agrícolas, año 2007. Madrid.

INE (2009). Encuesta Anual de Estructura Salarial. Madrid.

Ministerio de Trabajo (2010). Estadísticas e Informes de la Seguridad Social. Madrid.

Módenes, J.A. (2006). Movilidad espacial: uso temporal del territorio y poblaciones vinculadas. F.n. $X$ Congreso de la
Población Española: Migraciones, Movilidad y Territorio. Pamplona, AGE.

Nieto, A. y Gurría, J.L. (2008). Las políticas rurales europeas y su impacto en Extremadura. Boletín de la Asociación de Geógrafos Españoles, 48, p. 225-246.

Nieto, A. y Gurría, J.L. (2010). El modelo rural y el impacto de los Programas LEADER y PRODER en Extremadura (propuesta metodológica). Scripta Nova, XIV, 340, p. 57-70.

Oliva, J. (2006). Movilidad laboral y estrategias de arraigo rural. Revista Española de Estudios Agrosociales y Pesqueros, 211, p. 143-188.

Pérez Rubio, J.A. y Gurría Gascón, J.L. (2010). Neorrurales en Extremadura. Cáceres, Servicio de Publicaciones de la Universidad de Extremadura y Consejería de Agricultura y Desarrollo Rural.

TESYT (1991). Paro, mercado de trabajo y formación ocupacional en Extremadura. Mérida, Junta de Extremadura. 